\title{
Evaluation of a New Method for the Prevention of Neonatal Anemia
}

\author{
AMI BALIN, GIDEON KOREN, M. HASU, AND ALVIN ZIPURSKY \\ Divisions of Hematology/Oncology and Clinical Pharmacology, The Research Institute, The Hospital for Sick \\ Children, Toronto, Ontario M5G 1 X8, Canada
}

\begin{abstract}
Blood sampling for diagnostic purposes is a major cause of neonatal anemia. We propose a new method of blood sampling which preserves the infant's erythrocytes. Upon drawing of $1.5-\mathrm{ml}$ blood samples, the syringe is centrifuged and erythrocytes are injected back into the circulation. Using rabbits as an experimental model, we documented the efficiency of this method in decreasing the need for foreign blood transfusion. Results of a variety of laboratory tests performed with plasma obtained with the new method were identical to those achieved with whole blood. Subsequent testing did not show increased risk of contamination or bleeding phenomena secondary to heparin load. Red blood cells obtained by centrifugation were morphologically normal. This new method may be effective in reducing the need for foreign blood transfusions in neonates. (Pediatr Res 25:274-275, 1989)
\end{abstract}

Abbreviations

RBC, red blood cells

NZW, New Zealand white rabbits

One of the main causes of anemia in preterm infants is blood sampling for diagnostic purposes (1). Inevitably, preterm infants weighing less than $1500 \mathrm{~g}$ and whose blood vol is less than 120 $\mathrm{ml}$ have to be transfused with packed cells.

As a result of blood sampling for laboratory tests, some infants receive the equivalent of double or triple their blood vol in transfusions (1). Consequently, preterm infants are exposed to the adverse effects of blood transfusion, including infections (e.g. cytomegalovirus, hepatitis, AIDS), vol overload, mechanical erythrocyte injury, and alloimmunization (2).

We propose a new method of blood sampling which aims at reducing the demand for blood transfusion in newborn infants by preserving their erythrocytes during sampling.

\section{MATERIALS AND METHODS}

Rationale. For most laboratory tests, the plasma is needed, but the unused RBC are essential for the infant. The principle behind our proposal is to draw the infant's blood through an arterial line, to centrifuge it as a bedside procedure, and to return the erythrocytes to the infants while the plasma will be sent to the laboratory.

Proposed procedure. Samples of blood (1.0-1.5 ml) are drawn from an arterial line in a $2.5-\mathrm{ml}$ syringe, and the line is flushed as usual with infusion solution. The syringe is prepared with the appropriate anticoagulant (heparin, $50 \mu \mathrm{g} / 1$ of solution containing $1 \mathrm{U} / \mathrm{ml}$ ) according to the desired test. Subsequently, the syringe is introduced-together with a prepared water-filled sy-

Received April 4, 1988; accepted November 3, 1988.

Correspondence G. Koren, M.D., Division of Clinical Pharmacology, The Hospital for Sick Children, 555 University Avenue, Toronto, Ontario M5G 1X8, Canada. ringe as balance-to the centrifuge. The needle, positioned downward, is occluded by a rubber stopper. The centrifuge, preferentially located in the infant's room, should be quiet, fast, and with a short stopping time. We used the IEC 1450 centrifuge, clinical model IEC 428 . Optimally, the blood should be spun at 1200 $\operatorname{rpm}(200 \times g)$ for $3 \mathrm{~min}$. The disposable needle and stopper are then removed, and an 18- $\mu$ filter is attached (Hemo-Nate, with luer-lock hub, International Inc., San Antonio, TX). Subsequently, the erythrocytes, which are concentrated at the bottom of the syringe, are returned via the arterial line. The rate of transfusion should be $0.5 \mathrm{ml}$ in $5 \mathrm{~s}$ (3). The supernatant plasma is then transferred to a tube and sent to the laboratory.

To assess the efficacy and safety of this technique and to examine potential problems associated with its clinical use, the following experiments were conducted.

\section{EVALUATION OF TECHNIQUE}

Efficiency in preventing anemia. Two groups of adult NZW rabbits weighing approximately $2 \mathrm{~kg}$ were studied. All the rabbits were kept in separate cages and fed ad libitum. Their middle auricular artery was cannulated using a 24-gauge arterial catheter. Before the experiment, arterial blood was drawn for a complete blood count performed by Coulter Counter (Model S. plus IV, Coulter Electronics Inc., Hialeah, FL). Within $2 \mathrm{~min}, 18 \mathrm{ml}$ of blood were drawn in 12 syringes ( $1.5 \mathrm{ml}$ in each 3-cc syringe). This procedure was repeated twice daily for 2 consecutive $d$. The total amount of blood drawn from each animal was $72 \mathrm{ml}$ (the estimated blood vol of these rabbits is $90-140 \mathrm{ml}$ ) (4).

After each bleeding of $18 \mathrm{ml}$, the rabbits of the first group ( $n$ $=5$ ) were retransfused with their own packed cells as described above, and the animals of the second group (control group) $(n=$ 5) received the same quantity of normal saline ( $12 \mathrm{ml}$ for each bleeding episode, or a total of $48 \mathrm{ml}$ ). Complete blood counts were assessed in each rabbit at 1 and $16 \mathrm{~h}$ after the last bleeding episode.

Mean $\mathrm{Hb}$ level in the first group decreased by $17 \%$, from 123 $\pm 2.81 \mathrm{~g} / \mathrm{l}($ mean $\pm \mathrm{SE})$ to $99 \pm 1.75 \mathrm{~g} / \mathrm{l}$ at $16 \mathrm{~h}$ after the end of the study. In the control rabbits, there was a significantly $(p<$ 0.01 ) higher drop in $\mathrm{Hb}(48 \%$ ) (from $120 \pm 1.7 \mathrm{~g} / 1$ to $62 \pm 3.4$ $\mathrm{g} / \mathrm{l}$ ). (Fig. 1.) Reticulocyte count in the study group did not change (from $11.2 \pm 2.5 \%$ to $10.4 \pm 1.41$ ). In the control group, reticulocyte count significantly increased (from $5.7 \pm 0.13$ to $15.1 \pm 1.8)(p<0.01)$.

Reliability of laboratory tests performed on plasma obtained from proposed procedure. The aim of the following study was to determine whether laboratory tests are affected by the new procedure.

Blood samples of two human adults and four NZW rabbits served for this purpose. The rabbits (weighing $\sim 2 \mathrm{~kg}$ ) were cannulated in the middle auricular artery. A variety of laboratory tests was performed on samples of arterial blood and compared with samples of plasma obtained during our new procedure at the same time. In parallel, venous blood samples were sent in the same fashion to compare the results on "whole blood" to 
those of "centrifuge blood." The following tests were performed: glucose, urea, sodium, potassium, chloride, protein, albumin, total bilirubin, and glutamic oxaloacetic transaminase.

Arterial blood gases analysis $\left(\mathrm{pH}, \mathrm{P}_{\mathrm{CO}_{2}}, \mathrm{P}_{\mathrm{O}_{2}}\right.$, bicarbonate, and base excess) was carried out on samples from six NZW rabbits, one of them being ventilated with $\mathrm{Fi}_{\mathrm{O}_{2}}=1.0$. For all studies, the results from plasma obtained by the new method were identical to the regular method.

Potential bacterial contamination. To examine the possibility that the packed cells of the spun blood introduce bacteria to the blood circulation due to the proposed manipulation, the following studies were performed on 10 adult NZW rabbits. Two samples of blood were sent from each animal to the bacteriology laboratory: one sample was withdrawn from the arterial line as "whole blood," and the other was the packed cells portion after the described centrifugation of the blood. No antiseptic precautions were used. The samples were kept in aerobic and anaerobic conditions of culture for $7 \mathrm{~d}$ and then visualized for the existence of bacterial colonies.

Except for one sample of "whole blood" that grew aerobic bacteria colonies, no positive cultures were noted. None of the "packed cells" samples was contaminated.

In clinical practice, the risk of contamination, although small, is likely to increase with the number of times the arterial line must be entered. The proposed method requires two separate entries into the arterial line for each blood specimen instead of the usual one, and the actual additional risk of infections can only be assessed in a clinical trial.

Morphologic changes in $R B C$. The possibility that the new proposed procedure of blood sampling may alter erythrocyte morphology or lead to leukocyte or platelet aggregation was examined. After middle auricular artery cannulation in three NZW adult rabbits, arterial blood samples were collected and separated according to the described technique. In addition, venous blood samples of three human healthy adults were also tested in the same manner. From each sample, $50 \mu$ l of packed cells were removed and put in $0.3 \%$ glutaraldehyde for fixation (5). In addition, blood smears were prepared and stained with Wright's stain (6). Noncentrifuged blood samples from each subject served as controls-for glutaraldehyde-fixation and for stained smears. The examiner of the glutaraldehyde-fixed erythrocytes and the blood smears was not aware of the nature of the sample.

No alterations in the erythrocyte morphology were evident. No leukocyte or platelet aggregates could be identified in the "packed cells" portions of the samples.

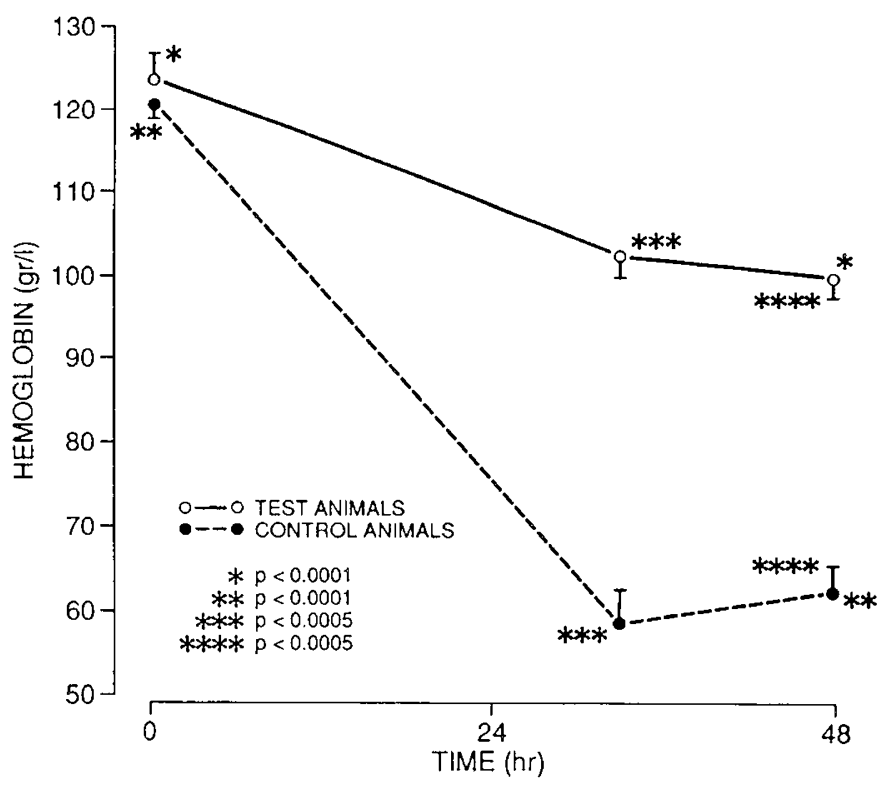

Fig. 1. Change in $\mathrm{Hb}$ in animals depleted of blood (control) versus those reinfused with their $\mathrm{RBC}$ according to the new method.
Heparinization of blood samples. The concentration of heparin in the syringes is expected to be high enough to prevent clotting of the blood while the maneuver takes place. Because part of the heparin is transfused back to the subjects with the packed cells, we defined the smallest amount of heparin needed to anticoagulate the blood. Prothrombin time and partial thromboplastin time (6) were tested on adult plasma mixed with various dilutions of heparin. The lowest concentration that prevented clotting of the plasma was $0.5 \mathrm{U}$ of heparin $/ \mathrm{ml}$ of blood.

The potential effect of heparin in a concentration of $1 \mathrm{U} / \mathrm{ml}$ on the coagulation system of rabbits in vivo was subsequently examined. In three NZW adult rabbits, a partial thromboplastin time test was performed on plasma before the procedure and 5 min after intraarterial administration of the packed cells portion.

No significant alterations in the partial thromboplastin time values were noted after the procedure. However, a clinical trial is needed to address the safety of heparinization of these samples in preterm infants. Moreover, our technique requires an additional flushing of the arterial line for each blood specimen withdrawn, and additional heparin is introduced into the circulation.

\section{DISCUSSION}

Although the risks of foreign blood transfusions have been long recognized, the emergence of AIDS has dramatically changed the perception of this problem. Preterm infants are different from adults in that the anemia is partially iatrogenic, stemming from repeated blood sampling from a very limited total vol (1). Because most blood tests are performed on serum or plasma, we have challenged the a priori assumption that RBC have to be discarded.

Our proposed technique may be effective in reducing the need for foreign blood transfusions. After loss of more than $50 \%$ of their blood vol, the adult NZW rabbits that received their own packed cells back had only $17 \%$ more reduction of their $\mathrm{Hb}$ level as opposed to the control animals, whose $\mathrm{Hb}$ values dropped by a mean of $48 \%$. The $17 \%$ reduction in $\mathrm{Hb}$ in the test group can be attributed to some use of erythrocytes for morphologic tests, but also to the incompleteness of the separation by the centrifuge. It is also theoretically possible that RBC survival is reduced by the new procedure, and this possibility will have to be addressed in future experiments. The reticulocytosis, seen in the first group and not in the second one, supports the concept of blood saving in the former and blood loss in the latter. Our subsequent studies revealed that this procedure does not alter laboratory data obtained from these samples.

The inexpensive and simple technique described accomplishes return to the patients of their own erythrocytes within minutes. No complications or side effects were seen in our animal model. The proposed method may reduce some of the serious risks associated with foreign blood transfusions.

In applying this method to infants, one will have to consider the issue of labeling and identification of the separated blood cells to assure that retransfusion of blood from another infant never takes place. The labor involved in labeling and identification will have to be added to the work associated in the new technique. Studies in newborn infants will assess the practicality of the method in clinical practice.

\section{REFERENCES}

1. Blancette VS, Zipursky A 1984 Assessment of anemia in newborn infants. Clin Perinatol 11:489

2. Blajchman MA, Sheridan D, Rawls WE 1984 Risks associated with blood transfusions in newborn infants. Clin Perinatol 11:403-415

3. Butt WW, Gow R, Whyte H, Smallhorn J, Koren G 1985 Complications resulting from use of arterial catheters retrograde flow and rapid elevation in blood pressure. Pediatrics 76:250-4

4. 1980 Guide to the care and use of experimental animals. Canadian Council on Animal Care, vol 1, Ottawa, Ont., p 86

5. Zipursky A, Brown E, Palko J, Brown EJ 1983 The erythrocyte differential count in newborn infants. Am J Pediatric Hematol Oncol 5:45-51

6. Wintrobe MM 1981 Principles of Hematologic Examination. In: Clinical Hematology, 8th ed. Lea \& Febiger, Philadelphia 